\title{
Modification of rheumatic symptoms by diet and drugs
}

\section{By Peter Merry, Bruce Kidd and David Blake, Inflammation Group, London Hospital Medical College, Turner Street, London E1 2AD}

Physicians and patients have long been intrigued with the possibility that some foods or food-related products may provoke rheumatic diseases, whereas others may alleviate symptoms of arthritis. If this hypothesis were true, then arthritis would respond to appropriate nutritional therapy. However, diet therapy for rheumatic disease has generally been considered a form of 'quack' therapy, for which a large percentage of patients with arthritis spend nearly $\$ 1$ billion annually in the USA (Panush, 1987). Surprisingly, despite the fervour of advocates and scepticism of rheumatologists, little objective information exists about nutritional therapy for rheumatic diseases, and virtually all conclusions have been based on inadequate information or improper study design (Ziff, 1983).

Persuasive reasons exist for considering that diet might affect rheumatic disease, and rheumatoid arthritis in particular. Two possible mechanisms suggested which need not be mutually exclusive are: (1) dietary antigens might provoke hypersensitivity responses (food allergies), which would in turn lead to rheumatological symptoms; (2) nutritional modifications might alter immune and inflammatory responses and thus affect manifestations of rheumatic diseases (Panush et al. 1986). We will continue by looking at these two concepts in more depth.

\section{Food-induced arthritis}

Gout is a rheumatic disease where the aetiological role of diet is established. Over 100 years ago the distinguished physician A. B. Garrod commented that 'fermented liquors' are a powerful predisposing cause of gout. More recently the purine content of the diet has been shown to influence the serum concentration of uric acid, the pathogenic moiety of gout (Seegmiller et al. 1961; Griebsch \& Zollner, 1974). The influence of diet in gout is so important, that the initial recommended prophylactic treatment is dietary manipulation, before embarking on lifelong drug therapy with allopurinol. What evidence is there of diet causing other rheumatic diseases?

A small number of tentative, yet provocative, observations suggest relationships between foods and rheumatic diseases. These include black walnut (Juglans nigra) ingestion with Behcet's syndrome (Marquardt et al. 1973), the case of a dermatologist who documented his own palindromic rheumatism to be caused by sodium nitrate hypersensitivity (Epstein, 1969), canavanine in lucerne (Medicago sativa) with SLE (Malinow et al. 1982), chemical or food challenges (particularly wheat, maize and beef) with symptomatic arthritis (Stroud, 1983), rheumatoid arthritis with dairy products (Parke \& Hughes, 1981), food and tartrazine sensitivity with rheumatoid arthritis (Brostoff, 1982; Wraith, 1982) and rheumatoid-like synovitis in rabbits with consumption of cow's milk (Reidenberg et al. 1983). How may foods be pathogenic in arthritic disease?

Although immunological mechanisms of tissue injury are important in the pathogenesis of rheumatic disease, the antigen or antigens that trigger these abnormal immune events have not been identified. Microbial agents have received a lot of attention, but food-related antigens should also be considered for the following reasons. Foods may normally evoke immune responses in humans, in the same way as other environmental antigens. Food antigens, food antibodies, and their complexes and sensitized lymphocytes have all been detected in the systemic circulation of normal subjects (Paganalli 
et al. 1979; Panush, 1986). Furthermore, some investigators have considered the gut to be more permeable to food antigens in patients with rheumatoid arthritis than in normal individuals (Sundqvist et al. 1982; Rooney et al. 1983). The role of antigenic absorption from the gut is further implied in the established phenomena of arthropathy following jejuno-ileal bypass (Delamere et al. 1983). Foods may cause immunologically-mediated symptoms after ingestion in some persons, symptoms that are usually anaphylactic, cutaneous, respiratory, or gastrointestinal and mediated by mechanisms of immediate hypersensitivity. A new concept of delayed reactions to foods (masked food intolerance) is emerging, in which it is hypothesized that certain clinical symptoms reflect food allergies that may develop after a few hours, days or even longer. It is proposed that this response is mediated by immunological mechanisms other than IgE-mast-cell events. These symptoms might include headaches, behavioural or gastrointestinal disorders, and arthritis (Panush, 1986).

Panush et al. (1986) have investigated a patient in depth, whose rheumatoid arthritis flared when dairy products were eaten. There was marked and consistent relief of these exacerbations (both subjective and objective) during fasting, which was sustained with elemental nutrition supplements. Four different blinded challenges with milk reproducibly exacerbated symptoms, whereas placebo and other food challenges had no effect. Symptoms peaked 24-28 h after each challenge and resolved after 1-3 d. Immunological studies suggested both delayed and immediate cutaneous reactivity to milk, no elevation of IgE anti-milk, marked increases of $\mathrm{IgG}$ and $\mathrm{IgG}_{4}$ anti-milk levels, marginally increased IgG-milk circulating immune complexes, and in vitro cellular sensitivity to milk. This study related symptomatic exacerbation of inflammatory arthritis with immunologic hypersensitivity to milk (Panush et al. 1986).

Animal experimental work has shown that nine out of twenty-five Old English rabbits drinking cow's milk for 12 weeks, developed rheumatoid-like synovial lesions. This was associated with a raised percentage of $T$ lymphocytes in the synovial fluid and high titres of serum and synovial fluid $\mathrm{Clq}$ binding activity, due to specific antibody $\mathrm{Clq}$ produced in response to C1q in cow's milk (Welsh et al. 1985a,b).

\section{Fasting and its effects on rheumatoid arthritis}

Impressive evidence for an effect of diet restriction on autoimmune disease was obtained by Fernandez et al. $(1976,1978)$ who showed that $(N Z B \times$ NZW) F1 'lupus' mice lived up to twice as long when total food intake was decreased. This was accompanied by a marked reduction in anti-DNA levels. It has also been demonstrated that the autoimmune disease of NZB mice is slowed by deprivation of zinc in the diet (Beach et al. 1981). Claims are sometimes made that fasting relieves arthritic symptoms in rheumatoid arthritis. It is known that malnutrition is immunosuppressive (Chandra, 1981), and studies have shown that fasting by healthy subjects is accompanied by altered neutrophil bacterial killing and depressed lymphocyte response to mitogens (Palmblad, 1976), decreased serum levels of acute-phase reactants and complement (Palmblad et al. 1977a) and increased serum cortisol concentrations (Palmblad et al. 1977b). In accordance with these findings, an association between improvement in inflammatory activity of rheumatoid joints, fall in the ESR and enhancement of neutrophil bactericidal capacity, following a $7 \mathrm{~d}$ fast has been reported (Uden et al. 1983). Skoldstrom et al. (1979) also noted symptomatic improvement of patients with rheumatoid arthritis during fasting.

\section{Rheumatoid arthritis and polyunsaturated fatty acids}

Adverse publicity surrounding the drugs used to treat rheumatoid arthritis and growing consumer enthusiasm for alternative medicine have increased interest in dietary 
manipulation as a form of therapy. Patients have long believed that fish oils in general, and cod liver oil in particular, may relieve arthritis. More recently it has been suggested that evening primrose (Oenothera biennis) oil may also be beneficial. What is the scientific evidence to support these claims?

Experimental evidence. Critical in the considerations involved in the possible effect of fatty acids on arthritis has been the amount of the 2-double-bonded polyunsaturated fatty acid (PUFA), linoleic acid (LA), in the diet. The level of intake of this fatty acid has a number of important ramifications above and beyond the fact that it is an essential fatty acid (EFA) whose absence from the diet can lead to deficiency states. First it is the precursor of arachidonic acid (AA) which is, in turn, the precursor of the prostaglandins (PG) via the cyclo-oxygenase pathway and leukotrienes via the 5-lipoxygenase (EC 1.13.11.12) pathway. Thus, decreased intake of LA leads to decreased formation of PG and leukotrienes, and increased intake of LA leads to increased amounts of these inflammatory agents. The E series PG (PGE) and leukotrienes have a pro-inflammatory effect (Kuehl \& Egan, 1980). The PGE also have important suppressive effects on both the cellular and humoral immune responses (Goodwin \& Webb, 1980) and on the response of the fibroblast to stimulation by immunologic mediators (Korn et al. 1980). So the PG may be expected to increase or decrease inflammation, immunity or collagen synthesis, depending on their site of action and their concentration at that site. As a corollary, diets which contain either large amounts of LA, leading to increased synthesis of PG, or small amounts of LA, leading to reduced PG synthesis, may be expected to affect inflammation, immunity and fibrosis.

There is a growing literature demonstrating the importance of the LA-AA-PG axis in inflammatory disease. Suppression of adjuvant arthritis in rats by injection of $P E_{2}$ (Aspinall \& Cammarata, 1969) and $\mathrm{PGE}_{1}$ (Zurier \& Quagliata, 1971) has been reported. Subsequent work demonstrated a beneficial effect of $P E_{1}$ on the survival of NZB/NZW mice (Zurier et al. 1977b) and on the course of renal disease in these mice (Zurier et al. 1977a). Kunkel et al. (1982) found that evening primrose oil, which is very rich in LA, markedly inhibited the development of adjuvant-induced arthritis in rats. Stackpoole \& Mertin (1981) showed that experimental allergic encephalomyelitis in guinea-pigs was almost completely inhibited by oral supplements of EFA. These reports have established the suppressive effects of PG or increased intake of PUFA on immunologically-mediated disease.

Conversely a number of studies have described benefits of EFA-deficient diets in animal models of immunologically-mediated inflammatory disease. Adjuvant arthritis was ameliorated in animals deficient in EFA (Denko, 1976). An EFA-deficient diet diminished humoral response to $T$ cell-dependent and $T$ cell-independent antigens in mice. Full restoration of these responses occurred on switching to a control diet (DeWille et al. 1978). Interestingly, Hurd et al. (1981) found that NZB/NZW mice fed on a diet in which all fat was in the form of saturated fat (coconut oil) had marked prolongation of life, and delay in the development of renal abnormalities. This improvement appeared to be associated with a deficiency of dietary PUFA. Conversely Prickett et al. (1981) reported that when NZB/NZW mice were fed on a diet rich in eicosapentaenoic acid (EPA) (the predominant fatty acid in the fish oil-rich diet of Greenland Eskimos), they experienced a similar marked prolongation of life and delay in onset of renal abnormalities. More recently Lee et al. (1985) showed that diets enriched with fish oil may have anti-inflammatory effects by inhibiting the 5-lipoxygenase pathway in neutrophils and monocytes, and by inhibiting the leukotriene $\mathrm{B}_{4}$-mediated function of neutrophils. McColl et al. (1986) reported that dietary supplementation with EPA reduced the severity of adjuvant arthritis in rats. There is, therefore, evidence that substitution of 
PUFA in the diet by saturated fatty acid or 'unphysiological' fish oil-derived PUFA, leads to a marked reduction in the severity of autoimmune disease in these mice.

Fish oils are rich in long-chain PUFA, particularly the $\omega-3$ series: EPA and docosahexaenoic acid. These are unlike LA which is an $\omega-6$ PUFA and is the predominant PUFA in the Western diet. One theory behind fish oil therapy is that fatty acids, such as $\alpha$-linolenic acid from fish oils, are converted into PG of the third series, whereas the saturated fats commonly consumed in the Western diet are converted via AA into PG of the second series. Because PG of the second series are actively involved in the rheumatoid process there may well be some benefit in diverting PG synthesis to the less inflammatory third series.

Thus, theoretically there appear to be three beneficial ways in which to modify fatty acid dietary intake in rheumatic diseases: (1) a PUFA-deficient diet aimed at reducing PG formation and decreasing lymphocyte and macrophage cell membrane reactivity. This diet could lead to the problem of EFA deficiency, so supplements may be required; (2) a diet rich in PUFA designed to increase the synthesis of supressor PG; (3) a diet rich in fish oils which would produce relatively inactive PG of the ' 3 ' series in place of the pro-inflammatory PG of the ' 2 ' series.

Clinical studies. Kremer et al. (1985) reported the first double-blind study to show that a diet high in PUFA and EPA in rheumatoid arthritis, led to improvement in morning stiffness, number of tender joints, and grip strength compared with controls who were eating an American diet high in saturated fats plus placebo EPA. These favourable results were not supported by laboratory markers of disease activity. More recently Kremer $e t$ al. (1987) studied the effects of supplementing an unchanged diet with fish oil supplements (15 'Max-EPA' (Duncan Flockhart) tablets/d) in rheumatoid arthritis. The leukotriene $B_{4}$ levels fell substantially and statistically significant improvement was seen in fatigue time and number of tender joints during the treatment period, but there was again no change in laboratory markers of disease activity. Sperling (1986) reported a synergistic effect of fish oils and non-steroidal anti-inflammatory drugs (NSAID) in rheumatoid arthritis in both clinical and biological indices.

It is believed that treatment with $\gamma$-linolenic acid from evening primrose oil, may produce a similar fall in PG of the second series as is found with fish oils, and also produce an increase in PG of the first series (e.g. $P G E_{1}$ ), which have anti-inflammatory activity. Hansen $e t$ al. (1983) found no benefit in twenty patients with active rheumatoid arthritis undergoing treatment with evening primrose oil for 12 weeks. Belch et al. (1986) investigated the effect both of evening primrose oil alone, and in combination with fish oil in rheumatoid arthritis. In contrast they found that both groups produced significant clinical improvement which allowed some patients to reduce or even stop their NSAID. They found no evidence of disease modification.

Many of these studies have been criticized for studying patients with early, often mild disease and response being clouded by hospitalization. Nash et al. (1988) studied twenty-six patients with well-established rheumatoid arthritis, treated as outpatients with E028 (an elemental diet consisting of essential amino acids, trace elements and vitamins, free of protein and considered hypo-allergic). A control group supplemented their diet with E028, whilst diet-treated patients used E028 alone for 4 weeks. After 4 weeks individual foods were re-introduced and disease activity was monitored. The results showed that this elemental diet improved functional score and a thermographic joint index, but not ESR or C-reactive protein.

The therapeutic implications of these studies may be somewhat disappointing to many rheumatologists because the quantitative changes were modest and reflected mainly subjective benefit. However, these observations are among the few that provide us with 
useful information about diet therapy, and point us in new directions for studying the pathogenesis of theumatic diseases and ultimately developing new therapies.

Another area of nutritional interest in rheumatic diseases which is open to therapeutic manipulation is iron. This is a major interest of our group.

\section{Fe and rheumatic disease}

The potential role of $\mathrm{Fe}$ in rheumatoid disease is well recorded (Blake $e t$ al. 1981). Any sustained inflammatory reaction causes changes in Fe metabolism, with a drop in serum $\mathrm{Fe}$ and a redistribution of Fe to the activated reticulo-endothelial system, including, in rheumatoid disease, the inflamed synovium. It has been suggested that this synovial Fe deposition contributes to the joint inflammation (Muirden \& Senator, 1968), and Blake et al. (1985a) found that high levels of synovial $\mathrm{Fe}$ anticipated a poor prognosis in early rheumatoid patients. Furthermore, treatment of the anaemia of chronic disease associated with rheumatoid arthritis, with intravenous Fe dextran (Blake et al. 1985b) or with oral Fe (Blake \& Bacon, 1982), has been shown to exacerbate joint symptoms.

The crucial role of $\mathrm{Fe}$ in inflammation is its ability to act as a redox agent, and hence take part in electron transfer pathways. Fe is thus able to catalyse the production of highly toxic reactive oxygen species (ROS) via the Fenton reaction. During inflammation it is thought that ROS produced by stimulated phagocytes contribute to tissue damage by inducing lipid peroxidation (Halliwell \& Gutteridge, 1984). Lipid peroxidation products have been found in synovial fluid both in rheumatoid patients (Lunec et al. 1981) and adjuvant arthritis (Yoshikawa et al. 1983), suggesting such a process may contribute to joint inflammation. High levels of $\mathrm{PGE}_{2}$ have been reported in inflamed tissue and perfusates of adjuvant arthritic ankle joints, and the levels measured reflected the severity (Barbier et al. 1984). Both periosteal proliferation and bone resorption may be induced by $\mathrm{PGE}_{2}$ (Galasko \& Bennett, 1976), and in vitro studies have shown that $\mathrm{Fe}$ nitrilotriacetate will augment the release of $P G E_{2}$ from synovial cells in culture (Okasaki et al. 1981). Fe chelation may, therefore, reduce $\mathrm{PGE}_{2}$ production, inhibiting both the periosteal reaction of adjuvant disease and the erosive consequences of synovial inflammation. It is interesting that this is an effect that can also be produced by dietary manipulation of fatty acids as mentioned earlier.

Manipulation of the diet by the removal of Fe thus has a sound theoretical basis for reducing the inflammation of rheumatoid arthritis. Andrews et al. (1987a) studied the effect of Fe chelation by using desferrioxamine (DFX) in adjuvant disease in rats. They found that DFX induced mild Fe deficiency, and reduced the incidence and severity of joint inflammation, but did not alter the local primary inflammatory response of adjuvant or the systemic sequelae. The joints showed a reduction in soft tissue swelling and bone erosion. These findings suggested an apparent selective influence of $\mathrm{Fe}$ on joint-mediated inflammation. They extended their studies to look at the effect of mild nutritional $\mathrm{Fe}$ deficiency on adjuvant disease in rats and also on models not involving the joint (carrageenan pleurisy, urate pleurisy, pyrophosphate foot pad; Andrews et al. 1987b). Mild nutritional Fe deficiency was found to significantly reduce the severity of adjuvant arthritis assessed by histology, radiology and subjective scoring. Again systemic features were unaffected, and there was no suppression of the other models of acute inflammation, supporting the selectivity of the phenomena. In Glynn-Dumonde synovitis of guinea-pigs, DFX stimulated the acute phase of inflammation, but repeated administration depressed the chronic phase (Blake et al. 1983).

These effects of DFX on chronic inflammation were sufficiently encouraging for preliminary trials on human rheumatoid patients. Of seven patients given $\operatorname{DFX}(3 \mathrm{~g} / \mathrm{d}, 5$ $\mathrm{d} /$ week for 1-3 weeks), four acquired retinal abnormalities that were reversed on drug 
withdrawal (Blake et al. 1985c). Also, two of the patients received the phenothiazine prochlorperazine during DFX therapy and subsequently lost consciousness for 48-72 h, possibly because this combination of drugs removes essential Fe from the nervous system. Thus there is a need for new Fe-chelating drugs that can be administered orally, and are safer than DFX.

\section{REFERENCES}

Andrews, F. J., Morris, C. J., Kondratowicz, G. \& Blake, D. R. (1987a). Annals of Rheumatic Diseases 46, 327-333.

Andrews, F. J., Morris, C. J., Lewis, E. J. \& Blake, D. R. (1987b). Annals of Rheumatic Diseases 46, 859-865.

Aspinall, R. L. \& Cammarata, P. S. (1969). Nature 224, 1320-1321.

Barbier, A., Navarro, J., Brehere, J. C. \& Roncucci, R. (1984). Agents and Actions 15, 103-110.

Beach, R. S., Gershman, M. E. \& Hurley, L. S. (1981). Journal of Immunology 126, 1999-2006.

Belch, J. J. F., Ansell, D., Madhok, R. \& Sturrock, R. D. (1986). British Journal of Rheumatology 25, Suppl., 75 Abstr.

Blake, D. R. \& Bacon, P. A. (1982). Lancet i, 623.

Blake, D. R., Gallagher, P. J., Potter, A. R., Bell, M. J. \& Bacon, P. A. (1985a), Arthritis and Rheumatism 27, 495-501.

Blake, D. R., Hall, N. D., Bacon, P. A., Dieppe, P. A., Halliwell, B. \& Gutteridge, J. M. C. (1981). Lancet ii, 1141-1144.

Blake, D. R., Hall, N. D., Bacon, P. A., Dieppe, P. A., Halliwell, B. \& Gutteridge, J. M. C. (1983). Annals of Rheumatic Diseases 42, 89-93.

Blake, D. R., Lunec, J., Ahern, M., Ring, E. F. J., Bradfield, J. \& Gutteridge, J. M. C. (1985b). Annals of Rheumatic Diseases 44, 183-188.

Blake, D. R., Winyard, P., Lunec, J. \& Williams, A. (1985c). Quarterly Journal of Medicine 56, 345-355.

Brostoff, J. (1982). Fourth Intemational Food Allergy Symposium of the American College of Allergy, Vancouver.

Chandra, R. K. (1981). British Medical Bulletin 37, 89-94.

Delamere, J. P., Baddeley, R. M. \& Walton, K. W. (1983). Annals of Rheumatic Diseases 42, 553-557.

Denko, C. W. (1976). Agents and Actions 6, 636-641.

DeWille, J. W., Fraker, P. J. \& Romsos, D. R. (1978). Journal of Nutrition 109, 1018-1027.

Epstein, S. (1969). Annals of Allergy 27, 343-349.

Fernandez, G., Friend, P., Yunis, E. J. \& Good, R. A. (1978). Proceedings of the National Academy of Sciences, USA 75, 1500-1504.

Fernandez, G., Yunis, E. J. \& Good, R. A. (1976). Proceedings of the National Academy of Sciences, USA 73, 1279-1283.

Galasko, C. S. B. \& Bennett, A. (1976). Nature 263, 508-510.

Goodwin, J. S. \& Webb, D. R. (1980). Clinical Immunology and Immunopathology 15, 106-122.

Griebsch, A. \& Zollner, N. (1974). Advances in Experimental Medicine and Biology 41, 443-449.

Halliwell, B. \& Gutteridge, J. M. C. (1984). Biochemical Journal 219, 1-14.

Hansen, T. M., Lerche, A., Kassis, V., Lorenzen, I. \& Sondergaard, J. (1983). Scandinavian Journal of Rheumatology 12, 85-88.

Hurd, E. R., Johnston, J. M., Okita, J. R., MacDonald, P. C., Ziff, M. \& Gilliam, J. N. (1981). Journal of Clinical Investigation 67, 467-485.

Korn, J. H., Haluska, P. V. \& LeRoy, E. C. (1980). Journal of Clinical Investigation 65, 543-554.

Kremer, J. M., Bigauoette, J. \& Michalek, A. V. (1985). Lancet i, 184-187.

Kremer, J. M., Jubiz, W. \& Rynes, R. I. (1987). Annals of Internal Medicine 106, 497-503.

Kuehl, F. A. \& Egan, R. W. (1980). Science 210, 978-984.

Kunkel, S. L., Ogawa, H., Ward, P. A. \& Zurier, R. B. (1982). Progress in Lipid Research 20, 885-888.

Lee, T. H., Hoover, R. L. \& Williams, J. D. (1985). New England Journal of Medicine 312, 1217-1224.

Lunec, J., Halloran, S. P., White, A. G. \& Dormandy, T. L. (1981). Journal of Rheumatology 8, 233-245.

McColl, S. R., Whitehouse, M. W., Cleland, L. G. \& Hurst, N. P. (1986). British Journal of Rheumatology 25, 106.

Malinow, M. R., Bardana, E. J., Pirofsky, B., Craig, S. \& McLoughlin, P. (1982). Science 216, 415-417.

Marquardt, J. L., Snyderman, R. \& Oppenheim, J. J. (1973). Cellular Immunology 9, 263-272.

Muirden, K. D. \& Senator, G. B. (1968). Annals of Rheumatic Diseases 27, 38-47. 
Nash, P., Workman, E., Smith, M., Hazleman, B. L. \& Hunter, J. O. (1988). British Journal of Rheumatology 27, Suppl. 1, 55 Abstr.

Okasaki, I., Brinckerhoff, C. E., Sinclair, J. F., Sinclair, P. R., Bronkowsky, H. L. \& Harris, E. D. (1981). Journal of Laboratory and Clinical Medicine 97, 396-402.

Paganalli, R., Levinsky, R. J., Brostoff, J. \& Wraith, D. G. (1979). Lancet i, 1270-1272.

Palmblad, J. (1976). Scandinavian Journal of Haematology 17, 217-226.

Palmblad, J., Cantell, K., Holm, G., Norberg, R., Strander, H. \& Sunblad, L. (1977a). Clinical and Experimental Immunology 30, 50-55.

Palmblad, J., Levi, L. \& Burger, A. (1977b). Acta Medica Scandinavica 201, 15-22.

Panush, R. S. (1986). Annals of Allergy 56, 500-503.

Panush, R. S. (1987). Annals of Internal Medicine 106, 619-621.

Panush, R. S., Stroud, R. M. \& Webster, E. M. (1986). Arthritis and Rheumatism 29, 220-226.

Parke, A. L. \& Hughes, G. R. V. (1981). British Medical Journal 282, 2027-2029.

Prickett, J. D., Robinson, D. R. \& Steinberg, A. D. (1981). Journal of Clinical Investigation 68, 556-559.

Reidenberg, M. M., Durant, P. J., Harris, R. A., Boccardo, G. P., Lahita, R. \& Stenzel, R. H. (1983). American Journal of Medicine 75, 365-370.

Rooney, P. J., Jenkins, R. T., Goodacre, R. L. \& Sivakumaran, T. (1983). Clinical Research 31, 160A.

Seegmiller, J. E., Grayzel, A. I., Laster, L. \& Liddle, L. (1961). Journal of Clinical Investigation 40, $1304-1314$.

Skoldstrom, L., Larsson, L. \& Linstrom, F. D. (1979). Scandinavian Journal of Rheumatology 8, 249-255.

Sperling, R. (1986). Advances in Rheumatology $1,8$.

Stackpoole, A. \& Mertin, J. (1981). Progress in Lipid Research 20, 649-654.

Stroud, R. M. (1983). In Current Topics in Rheumatology, pp. 145-157 [B. H. Hahn, F. C. Arnett, T. M. Zizic and M. C. Hochberg, editors]. Kalamazoo, MI: Upjohn Press.

Sundqvist, T., Linstrom, F., Magnusson, K. E., Skoldstrom, L., Stjernstom, I. \& Tagesson, C. (1982). Scandinavian Journal of Rheumatology 11, 33-38.

Uden, A. M., Trang, L., Venizelos, N. \& Palmblad, J. (1983). Annals of Rheumatic Diseases 42, 45-51.

Welsh, C. J. R., Hanglow, A. C., Conn, P., Barker, T. H. W. \& Coombs, R. R. A. (1985a). International Archives of Allergy and Applied Immunology 78, 145-151.

Welsh, C. J. R., Hanglow, A. C., Conn, P., Barker, T. H. W. \& Coombs, R. R. A. (1985b). International Archives of Allergy and Applied Immunology 78, 152-157.

Wraith, D. G. (1982). Fourth International Food Allergy Symposium of the American College of Allergy, Vancouver.

Yoshikawa, T., Tanaka, H. \& Kondo, M. (1983). Journal of Applied Biochemistry 5, 382-387.

Ziff, M. (1983). Arthritis and Rheumatism 26, 457-461.

Zurier, R. B., Damjanov, I., Sayadoff, D. M. \& Rothfield, N. F. (1977a). Arthritis and Rheumatism 20, $1449-1456$.

Zurier, R. B., Sayadoff, D. M., Torrey, S. B. \& Rothfield, N. F. (1977b). Arthritis and Rheumatism 20, 723-728.

Zurier, R. B. \& Quagliata, F. (1971). Nature 234, 304-305. 